\title{
New-Onset Refractory Status Epilepticus: More Investigations, More Questions
}

\author{
Philippe Dillien $^{a} \quad$ Susana Ferrao Santos ${ }^{b, c}$ Vincent van Pesch ${ }^{b, c}$ \\ Vanessa Suin $^{d}$ Sophie Lamoral $^{d}$ Philippe Hantson $^{\mathrm{a}}$ \\ ${ }^{a}$ Department of Intensive Care, Cliniques St-Luc, Université catholique de Louvain, \\ Brussels, Belgium; ${ }^{b}$ Department of Neurology, Cliniques St-Luc, Université catholique de \\ Louvain, Brussels, Belgium; 'Laboratory of Neurophysiology, Cliniques St-Luc, \\ Université catholique de Louvain, Brussels, Belgium; ${ }^{d}$ Viral Diseases Unit, Scientific \\ Institute of Public Health (WIV-ISP), Brussels, Belgium
}

\section{Keywords}

New-onset refractory status epilepticus · Cryptogenic origin · Seronegative limbic encephalitis - Japanese encephalitis virus · SMC3 gene

\begin{abstract}
A 27-year-old previously healthy woman was admitted to the hospital with recurrent seizures. Status epilepticus developed that became refractory to third-line therapy with propofol and barbiturates. The patient had a very extensive diagnostic workup including autoimmune, viral and genetic investigations. A tentative immune therapy was proposed with high doses of steroids and plasma exchanges. Our patient had an inherited heterozygous single nucleotide variant in the sequence c.1280A $>$ G [p.Lys427Arg] of the SMC3 gene that was insufficient to explain the seizures. Surprisingly, IgM antibodies against Japanese encephalitis virus were positive on the serum drawn 11 days after symptom onset, as detected by ELISA and the immunofluorescence antibody (IFA) technique. IgG antibodies were also positive using the IFA technique, but not with ELISA. The same investigations as well as the detection of the viral genome by the q-RT-PCR technique were negative on cerebrospinal fluid. Despite the suspicion of a viral infection, we concluded that our patient had a new-
\end{abstract}




\section{Case Reports in Neurology}

Dillien et al.: New-Onset Refractory Status Epilepticus: More Investigations, More Questions

onset refractory status epilepticus of cryptogenic origin. Termination of the status epilepticus was obtained after 47 days, with a possible benefit from the introduction of ketamine.

(C) 2016 The Author(s)

Published by S. Karger AG, Basel

\section{Introduction}

Previously healthy adults may present with status epilepticus (SE) that becomes rapidly refractory to standard first- and second-line agents. Despite extensive workup, no clear cause can be found in some cases. This entity is now often defined as cryptogenic new-onset refractory status epilepticus (NORSE) and usually carries a poor prognosis [1]. However, few cases with a favourable outcome have been reported. It also appears likely that some infectious, autoimmune or genetic aetiologies may be misdiagnosed [2]. The optimal management for this devastating disorder remains unclear. We describe a case with a favourable outcome and discuss how the extensive investigations for rare aetiologies can be either not fully conclusive or even puzzling.

\section{Case Report}

A 27-year-old woman (weight: $50 \mathrm{~kg}$; height: $170 \mathrm{~cm}$ ) was admitted to the hospital for palpebral myoclonus and 'eye-rolling' episodes with impairment of consciousness. Her past medical history was not relevant. The patient had traveled to Asia more than 4 months before symptom onset. She complained of a flu-like syndrome 1 week before hospital admission. Tonic-clonic seizures were observed soon after hospital admission and were refractory to benzodiazepine administration. A standard electroencephalogram (EEG) showed focal ictal discharges with predominant right hemispheric onset, but occasional discharges with left onset were also observed. Brain MRI performed soon after admission failed to reveal any lesion. Cerebrospinal fluid (CSF) analysis showed low cellularity ( $<10$ cells/ $\mu$ l), with normal glucose, protein and lactate concentrations. No CSF-specific IgG oligoclonal bands were found upon immunoelectrophoresis.

As mechanical ventilation was required, the patient was transferred to the intensive care unit (ICU) (day 1) for the pharmacological treatment of SE that included benzodiazepines and sodium valproate, which were rapidly shifted to phenytoin and levetiracetam. Persistent seizures lead to the prescription of general anaesthetics: on day 1, midazolam and propofol, which was replaced by thiopental on day 3 , after the ineffective introduction of ketamine on day 2 (administered as a bolus of $1 \mathrm{~m} / \mathrm{kg}$, followed by a continuous infusion of $2.5 \mathrm{mg} / \mathrm{kg} / \mathrm{h}$ ). Additionally, as an autoimmune encephalitis was considered a possible diagnosis, the patient received a tentative 5-day course of high-dose (1 g) intravenous methylprednisolone (days 2-6) followed by 2 courses of plasma exchange (days 5 and 7). The basic antiepileptic regimen was shifted to lacosamide and topiramate on day 12 (up to $400 \mathrm{mg} /$ day for both drugs). Magnesium infusion (days 14-20) was also found ineffective. A ketogenic diet was unsuccessfully tried from day 16 and stopped after 2 weeks. Despite increasing doses of thiopental (up to $300 \mathrm{mg} / \mathrm{h}$ ), a rapid tolerance developed. While the patient appeared fully sedated, electrical seizures with right temporo-occipital onset were still observed (fig. 1). After the reintroduction of high doses of propofol ( $400 \mathrm{mg} / \mathrm{h}$ on day 27) in replacement of thiopental, sustained episodes of 'burst suppression' were obtained, while bilateral independent periodic discharges remained (fig. $2 a$, b). On hospital day 33, it was 
decided to reintroduce ketamine at a higher infusion rate $(5 \mathrm{mg} / \mathrm{kg} / \mathrm{h}$, after a bolus of 2 $\mathrm{mg} / \mathrm{kg}$ ) for a period of 2 days. The patient was still receiving propofol $(10 \mathrm{mg} / \mathrm{kg} / \mathrm{h})$, but midazolam was withdrawn. EEG showed the reappearance of a 5- to 6- $\mathrm{Hz}$ irregularly shaped basal activity, mixed with diffuse beta activity. The patient showed an improved reactivity (fig. 2c). However, after $48 \mathrm{~h}$ of ketamine infusion, there were episodes of electrical seizures with bilateral discharges of high-voltage fast activity (fig. 2d). Clinical generalized convulsive seizures reappeared. Carbamazepine was ultimately introduced. The termination of electrical SE was determined on day 47. The patient was discharged from the ICU on day 53. Her consciousness state gradually improved. The results of the investigations performed during the ICU stay for uncommon aetiologies of SE may be summarized as follows.

There was no laboratory evidence for autoimmune encephalitis. Indeed, both CSF and serum samples were ultimately negative for the following antibodies (indirect immunofluorescence biochip mosaic assay; Biognost, Heule, Belgium): NMDAR, DPPX, CASPR2, LGI1, GABA-b, amphiphysin, CV2, PNMA2/Ta, Ri, Yo, Hu, Recoverin, SOX1, Titin, Zic4, GAD65, and DNER.

Common causes of viral encephalitis were also rapidly excluded. The results of more specialized virological investigations (flavivirus-mediated encephalitis, such as dengue and yellow fever, tick-borne encephalitis and Japanese encephalitis) were received with delay. These analyses were performed in the Viral Diseases Unit of the Scientific Institute of Public Health (WIV-ISP). IgM antibodies against Japanese encephalitis virus (JEV) were positive on the serum drawn 11 days after symptom onset, using the Anti-Japanese Encephalitis Virus Elisa IgM kit (Euroimmun) and the immunofluorescence antibody (IFA) technique Biochip Flavivirus Mosaic 3 (IgG/IgM; Euroimmun); IgG antibodies were also positive as detected by IFA technique, but not by ELISA. The same investigations as well as the detection of the viral genome by q-RT-PCR technique were negative on the CSF [3]. However, on the late control samples obtained 5 months later, IgM and IgG antibodies were negative with both ELISA and IFA techniques. Serological analysis for the dengue virus using the Anti-Dengue Virus Elisa IgM/IgG kit (Euroimmun), the West Nile virus using the IFA technique Biochip Flavivirus Mosaic 3 (IgG/IgM; Euroimmun) and the tick-born encephalitis virus using the Immunozym FSME IgG/IgM Elisa kit (Progen) as well as the seroneutralization test were all negative [4]. Virological analysis for dengue and yellow fever as well as for tick-borne encephalitis virus was also negative.

With respect to genetic aetiologies of epilepsy, POLG mutations were excluded. However, next-generation sequencing revealed a heterozygous single nucleotide variant in the sequence c. $1280 \mathrm{~A}>\mathrm{G}$ [p.Lys427Arg] of the SMC3 gene. The substitution was inherited from her father, who is not symptomatic.

A brain positron emission tomography (PET) with $18 \mathrm{~F}$-fluorodeoxyglucose was performed on day 43 and showed a right hippocampal hypermetabolism, while total-body FDGPET was negative. The diagnosis of seronegative limbic encephalitis was suggested. A followup brain PET scan performed 3 months after symptom onset showed almost complete regression of the right hippocampal hypermetabolism. Late brain MRI (day 93) revealed moderate bilateral right predominant hippocampal atrophy. Total body ${ }^{18} \mathrm{~F}$-fluorodeoxyglucose PET was not relevant.

At the 6-month follow-up, the patient had persisting attention and memory deficits and mild impairment of executive functions, but had regained almost complete autonomy for daily life tasks. The patient is still presenting some temporal (2/week) and opercular $(2 /$ month) seizures, as well as rare secondary generalized tonic-clonic seizures. Some improvement was noted after the introduction of lamotrigine. 


\section{Discussion}

This young, previously healthy woman, without a prior history of epilepsy, developed super-refractory SE following a mild febrile illness, and no identifiable cause was found. This entity is now often defined as cryptogenic NORSE [5]. The absence of a proven aetiology was mandatory in the early series of NORSE, but the definition of 'cryptogenic' is evolving continuously as new aetiologies, mostly autoimmune, of refractory SE are discovered. In a recent retrospective analysis of 130 cases collected between 1 January 2008 and 31 December 2013 in 13 academic medical centres, 67 (52\%) remained cryptogenic, and the most common aetiologies were autoimmune (19\%) and paraneoplastic (18\%) encephalitis [6]. It appears likely that both aetiologies are probably underestimated due to variable diagnostic workup. This is probably true for autoimmune encephalitis with novel autoantibodies yet to be identified. As mentioned in the case description, a very extensive investigation of possible autoimmune aetiologies, with negative results, was performed in this case. Clinically, it is impossible to differentiate cryptogenic cases from autoimmune cases. This is also the case for CSF investigations or brain imaging. The brain PET scan which was performed more than 1 month after symptom onset demonstrated hypermetabolism in the right hippocampal area. This was the possible anatomical substrate for seronegative limbic encephalitis. In a recent series of 18 patients with non-infectious limbic encephalitis and explored by FDGPET, 9 had antibodies against surface antigens, 5 against intracellular antigens, and 5 no autoantibodies [7]. Four of the 5 antibody-negative patients showed mesiotemporal hypermetabolism on FDG-PET. In comparison with the patients who had antibodies against intracellular antigens and rather similar FDG-PET findings, the authors speculated that in antibody-negative patients, yet to be identified antibodies would also be directed against intracellular antigens or would produce an inflammatory reaction similar to that of known intracellular autoantibodies.

Viral aetiologies of refractory SE remain extremely rare, when the common viruses can be excluded. Extensive viral investigations were performed in this case, including testing for the West Nile virus, yellow fever, dengue and tick-borne encephalitis viruses. We found IgM and IgG antibodies in the serum which recognized JEV antigens in the IFA test. In the absence of a JEV vaccination history, this might be due to (previous) infection with JEV or an unknown virus which is antigenically related to JEV. However, there are two issues which do not corroborate the hypothesis of a JEV infectious aetiology: no antibodies against JEV were detected in the CSF, and no IgG antibodies could be detected by ELISA. The disappearance of IgM and IgG antibodies on the late serum samples could be the consequence of the plasma exchanges performed.

No case of JEV has been described so far in Belgium or Europe, except for imported cases. Nevertheless, a national mosquito survey (MODIRISK) has revealed the introduction of an invasive mosquito species, Aedes j. japonicus, in Belgium since 2002, first located in a secondhand tire company in a small village at a 20-km distance from our patient's home [8, 9].

Finally, our patient had an inherited heterozygous single nucleotide variant in the sequence c. $1280 \mathrm{~A}>\mathrm{G}$ [p.Lys427Arg] of the SMC3 gene. While mutations in the SMC3 gene have been associated with the Cornelia de Lange syndrome type 3, our patient had no expressive phenotype, and the clinical significance of the present variant remains unknown and insufficient to explain NORSE [10].

The treatment of NORSE remains challenging and the disease usually carries a poor prognosis. Two strategies applied in the present case may be debatable. The use of anaesthetics tends to be associated with a poor outcome, higher complication rates and longer SE 


\section{Case Reports in Neurology}

duration [11]. The risk increases with the number of anaesthetics used either sequentially or concomitantly. However, after adjusting for confounders, the severity of refractory SE itself is the main determinant of a poor outcome, rather than the use of anaesthetics [12]. The use of ketamine in NORSE is poorly documented $[13,14]$. Ketamine is widely believed to act principally through the non-competitive antagonism of N-methyl-D-aspartate (NMDA) receptor mediated glutamatergic activity, while propofol is considered as a prominent $\gamma$ aminobutyric acid receptor type A (GABA-a) agonist. To date, the electroencephalographic effects of the combination of high doses of propofol and ketamine have been poorly investigated. On its own, ketamine increases theta (4-8 Hz) and decreases alpha (8-13 Hz) oscillations, whereas in the presence of propofol, ketamine induces a significant shift to beta band frequencies (13-30 Hz) [15]. It has recently been described that such modification of the EEG background constitutes a prognostic factor for refractory SE termination [15]. Interestingly, in our case, the first ketamine infusion did not achieve a modification of the background activity, while the second infusion brought a shift towards theta-beta activity, soon before refractory SE termination. The interpretation of the specific ketamine effect could be biased by a concurrent midazolam withdrawal, which could also account for the observed clinical and electrical modifications. Due to drug interactions or combined toxicities, concomitant drug changes are inevitable in such severe cases of SE, undermining our understanding of clinically relevant observations outside the set of clinical trials.

Finally, our patient received early immune therapy with high-dose steroids and plasma exchanges, contrasting to the general strategy for cryptogenic NORSE [16]. It seems impossible to determine if this therapy contributed to the termination of SE or to the final favourable outcome.

In conclusion, despite extensive diagnostic workup including autoimmune, viral and genetic investigations, the definitive cause of NORSE remained unidentified in our patient. Unexpected findings even complicated the case discussion without any retrospective influence on the management. The role of some specific anaesthetics like ketamine and of early immune therapy in cryptogenic NORSE warrants further investigation.

\section{Statement of Ethics}

Informed consent was obtained from the patient.

\section{Disclosure Statement}

The authors declare that there is no conflict of interest regarding the publication of this paper.

\section{References}

1 Costello DJ, Kilbride RD, Cole AJ: Cryptogenic new onset refractory status epilepticus (NORSE) in adults - infectious or not? J Neurol Sci 2009;277:26-31.

2 Glaser CA, Gilliam S, Honarmand S, Tureen JH, Lowenstein DH, Anderson LJ, Bollen AW, Solbrig MV: Refractory status epilepticus in suspect encephalitis. Neurocrit Care 2008;9:74-82.

3 Patel P, Landt O, Kaiser M, Faye 0, Koppe T, Lass U, Sall AA, Niedrig M: Development of one-step quantitative reverse transcription PCR for the rapid detection of flaviviruses. Virol J 2013;10:58 
Dillien et al.: New-Onset Refractory Status Epilepticus: More Investigations, More Questions

4 Roelandt S, Suin V, Riocreux F, Lamoral S, Van der Heyden S, Van der Stede Y, Lambrecht B, Caij B, Brochier B, Roels S, Van Gucht S: Autochtonous tick-borne encephalitis virus seropositive cattle in Belgium: a risk-based serological survey. Vector Borne Zoonotic Dis 2014;14:640-647.

5 Gaspard N, Foreman BP, Alvarez V, Cabrera Kang C, Probasco JC, Jongeling AC, Meyers E, Espinera A Haas KF, Schmitt SE, Gerard EE, Gofton T, Kaplan PW, Lee JW, Legros B, Szaflarski JP, Westover BM, LaRoche SM, Hirsch LJ; Critical Care EEG Monitoring Research Consortium (CCEMRC): New-onset refractory status epilepticus. Neurology 2015;85:1604-1613.

$6 \quad$ Najjar S, Pearlman D, Devinsky 0, Najjar A, Nadkarni S, Butler T, Zagzag D: Neuropsychiatric autoimmune encephalitis without VGKC-complex, NMDAR, and GAD autoantibodies: case report and literature review. Cogn Behav Neurol 2013;26:36-49.

7 Baumgartner A, Rauer S, Mader I, Meyer PT: Cerebral FDG-PET and MRI findings in autoimmune limbic encephalitis: correlation with autoantibody types. J Neurol 2013;260:2744-2753.

8 Versteirt V, Schaffner F, Garros C, Dekoninck W, Coosemans M, Van Bortel W: Introduction and establishment of the exotic mosquito species Aedes japonicus japonicus (Diptera: Culicidae) in Belgium. J Med Entomol 2009;46:1464-1467.

9 Damiens D, Ayrinhac A, Van Bortel W, Versteirt V, Dekoninck W, Hance T: Invasive process and repeated cross-sectional surveys of the mosquito Aedes japonicus japonicus establishment in Belgium. PLoS One 2014;9:e89358.

10 Pavlidis E, Cantalupo G, Bianchi S, Piccolo B, Pisani F: Epileptic features in Cornelia de Lange syndrome: case report and literature review. Brain Dev 2014;36:837-843.

11 Shorvon S, Ferlisi M: The outcome of therapies in refractory and super-refractory status convulsive status epilepticus and recommendations for therapy. Brain 2012;135:2314-2328.

12 Marchi NA, Novy J, Faouzi M, Stöhli C, Burnand B, Rossetti AO: Status epilepticus: impact of therapeutic coma on outcome. Crit Care Med 2015;43:1003-1009.

13 Zeiler FA, Teitelbaum J, Gillman LM, West M: NMDA antagonists for refractory seizures. Neurocrit Care 2014;20:502-513.

14 Gaspard N, Foreman B, Judd LM, Brenton JN, Nathan BR, McCoy BM, Al-Otaibi A, Kilbride R, Fernández IS, Mendoza L, Samuel S, Zakaria A, Kalamangalam GP, Legros B, Szaflarski JP, Loddenkemper T, Hahn CD, Goodkin HP, Claassen J, Hirsch LJ, Laroche SM: Intravenous ketamine for the treatment of refractory status epilepticus: a retrospective multicenter study. Epilepsia 2013;54:1498-1503.

15 Basha MM, Alqallaf A, Shah AK: Drug-induced EEG pattern predicts effectiveness of ketamine in treating refractory status epilepticus. Epilepsia 2015;56:e44-e48.

16 Khawaja AM, DeWolfe JL, Miller DM, Szaflarski JP: New-onset refractory status epilepticus (NORSE): the potential role for immunotherapy. Epilepsy Behav 2015;47:17-23. 


\section{Case Reports in Neurology}

Case Rep Neurol 2016;8:127-133

$10.1159 / 000447295$

(C) 2016 The Author(s). Published by S. Karger AG, Basel www.karger.com/crn

Dillien et al: New-Onset Refractory Status Epilepticus: More Investigations, More Questions

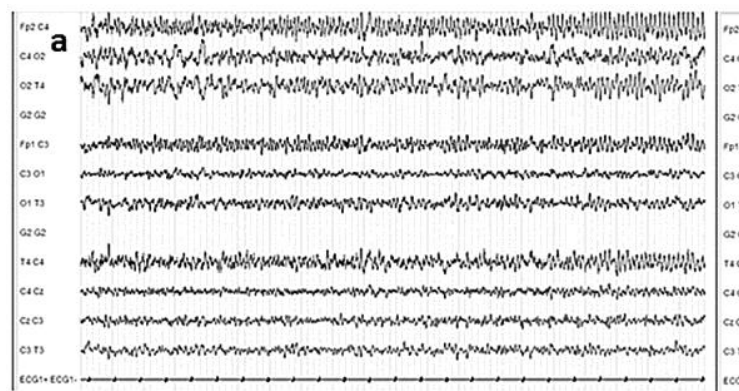

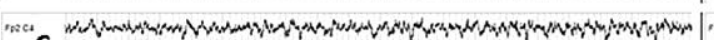

${ }_{\text {Ca: }} \mathrm{C}$,

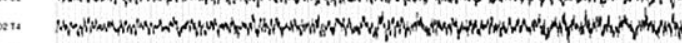

oras$$
\text { cor }
$$$$
\text { aras }
$$$$
\text { cace }
$$

Fig. 1. Continuous EEG recordings showing a right-sided electrical seizure, starting with temporo-occipital low-voltage fast activity $(\mathbf{a}, \mathbf{b})$ followed by bilateral central sharp waves $(\mathbf{c}, \mathbf{d})$.

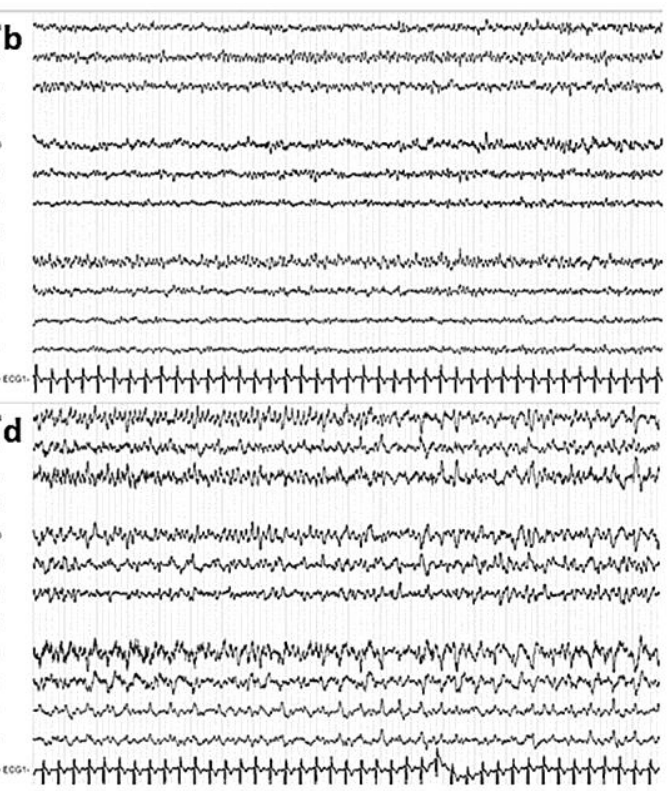

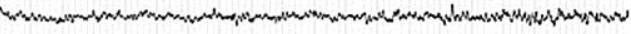

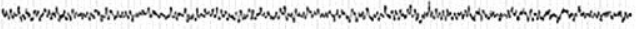

Hint Fis:

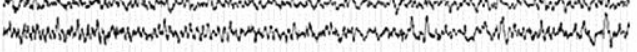

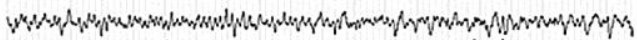

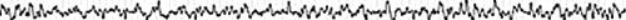

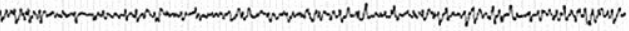

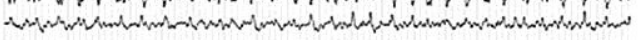

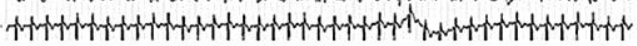
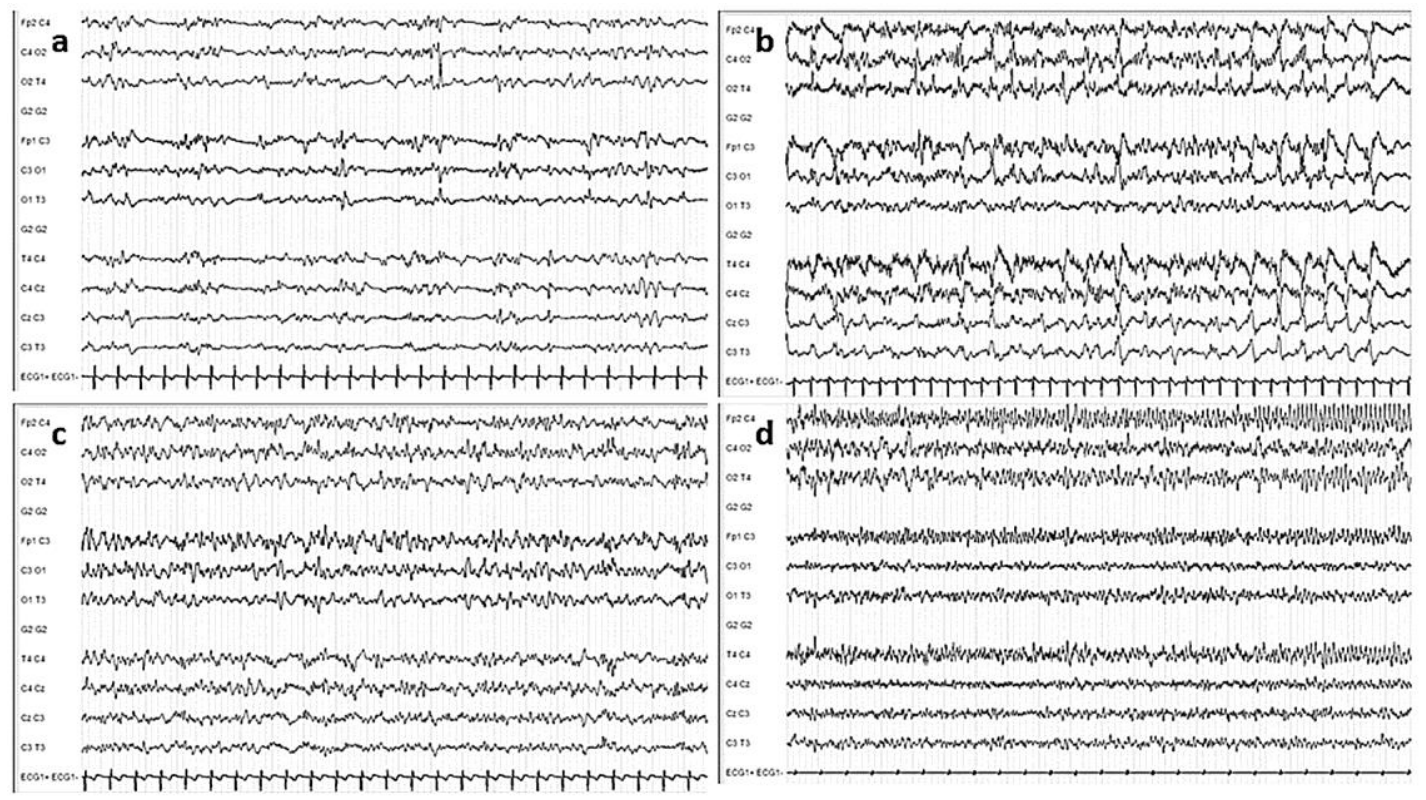

Fig. 2. Continuous EEG recordings showing, under high-dosage propofol and midazolam infusion, a typical burst-suppression pattern, with persistent bilateral independent periodic discharges (a, b). After the second ketamine infusion, theta-beta activity replaced the burst-suppression background pattern (c). Recordings show the reoccurrence of electrical seizures with bilateral discharges of high-voltage fast activity (d). 